\title{
Homens lusófonos, consumo de álcool e riscos de câncer: Sob a influência cultural da
}

\section{masculinidade}

\author{
Portuguese-speaking men, alcohol consumption and cancer risks: Under the cultural influence of \\ masculinity
}
Hombres de habla portuguesa, consumo de alcohol y riesgos de cáncer: Bajo la influencia cultural de la masculinidad

Recebido: 26/05/2021 | Revisado: 04/06/2021 | Aceito: 08/06/2021 | Publicado: 22/06/2021

\author{
Margareth Santos Zanchetta \\ ORCID: https://orcid.org/0000-0003-2321-9438 \\ Ryerson University, Canadá \\ E-mail: mzanchet@ ryerson.ca \\ Ingryd Cunha Ventura Felipe \\ ORCID: https://orcid.org/0000-0002-8340-307X \\ Ryerson University, Canadá \\ E-mail: ingrydventura@gmail.com \\ Renê Spezani \\ ORCID: https://orcid.org/0000-0002-5347-6112 \\ Centro Universitário Augusto Motta, Brasil \\ E-mail: renespezani@gmail.com \\ Veronica Finamore \\ ORCID: https://orcid.org/0000-0003-0520-9519 \\ Ryerson University, Canadá \\ E-mail: finamore.veronica@gmail.com \\ Christian Bergeron \\ ORCID: https://orcid.org/0000-0002-1079-4508 \\ University of Ottawa, Canadá \\ E-mail: cberger9@uottawa.ca
}

\begin{abstract}
Resumo
Objetivo- Explorar as percepções culturais subjacentes ao conceito de masculinidade sobre o consumo de álcool e o risco potencial de câncer por homens lusófonos. Metodologia- Sondagem quanti-qualitativa anônima online pela plataforma Opinio (Maio 2018-Março 2019). Resultados- Respondentes vivendo no Brasil, Canadá, Portugal e USA $(\mathrm{n}=184)$ indicaram que o consumo do álcool integra a identidade cultural masculina principalmente como percebida por $89 \%$ dos respondentes brasileiros. A ocupação profissional de maior tendência para o consumo do álcool é a construção civil $(23.2 \%)$ e seu consumo foi percebido por $17.5 \%$ dos respondentes como sendo parte da vida dos estudantes masculinos, enquanto que o desemprego ou a instabilidade no trabalho foram relatados por $17.5 \%$ como relacionados ao contexto de vida masculina. A análise temática foi guiada pelos temas: Práticas particulares do consumo alcoólico segundo a percepção de masculinidade no contexto etnocultural lusófono; e, Tendências do consumo alcoólico influenciado pela origem etnocultural, pressão social, e resposta aos fatores estressantes como risco potencial para o câncer. Conclusão- Respondentes recomendaram a maior acessibilidade dos resultados de pesquisa demonstrando a relação entre consumo de álcool e maior risco para o desenvolvimento de câncer como fator de desmistificação da inocuidade do álcool em relação ao câncer. A pesquisa multidisciplinar e aquela conjunta entre a Enfermagem do Trabalho e a Oncológica podem redirecionar políticas preventivas do câncer aos homens. Esforços futuros devem incluir ações tanto no âmbito individual como no coletivo, com medidas para atuação sobre os fatores de risco que podem aumentar o risco de câncer.
\end{abstract}

Palavras-chave: Comportamentos de risco à saúde; Consumo de bebidas alcoólicas; Masculinidade; Neoplasias; Saúde do homem.

\begin{abstract}
Objective- To explore the cultural perceptions of masculinity in relation to alcohol consumption and its potential risk of cancer by Portuguese-speaking men. Method- An online anonymous quanti-qualitative survey hosted by the Opinio platform (May 2018-March 2019). Results- Participants living in Brazil, Canada, Portugal and USA ( $n=184$ ) indicated that alcohol consumption is integrated in men's cultural identity, primarily as perceived by $89 \%$ of Brazilian respondents. The professional occupation reported as most susceptible to alcohol consumption is civil construction $(23.2 \%), 17.5 \%$ of respondents reported alcohol as being part of the lives of students, and unemployment or instability
\end{abstract}


at work were reported by $17.5 \%$ as reasons for alcohol consumption in men. Thematic analysis was guided by the following themes: Particular practices of alcoholic consumption according to the perception of masculinity in the Portuguese-speaking ethnocultural context; and, Trends in alcohol consumption influenced by ethnocultural origin, social pressure, and response to stressful factors, as potential risk to cancer. Conclusion- Participants recommended widespread availability of research results demonstrating the relationship between alcohol consumption and risk for cancer as a factor in demystifying alcohol use in relation to cancer. Multidisciplinary and joint research between Occupational and Oncology Nursing can redirect preventive cancer policies to men. Future efforts should include actions both at the individual and collective level, with measures to act on risk factors that may increase the risk of cancer.

Keywords: Alcohol drinking; Health risk behaviours; Masculinity; Men’s health; Neoplasms.

\section{Resumen}

Objetivo -Explorar las percepciones culturales subyacentes bajo el concepto de la masculinidad con el consumo de alcohol y los riesgos potenciales de cáncer en los hombres de habla portuguesa. Metodología- Encuesta cuanticualitativa anónima en línea desde la plataforma Opinio (Mayo de 2018 a Marzo de 2019). Resultados- Participantes en Brasil, Canadá, Portugal y EE.UU. $(n=184)$ indicaron que el consumo de alcohol es parte de la identidad cultural masculina, principalmente percibida por los participantes brasileños (89\%). La ocupación profesional de mayor tendencia al consumo de alcohol es la construcción civil $(23.2 \%)$, y su consumo fue percibido por $17.5 \%$ de los encuestados como parte de la vida de los estudiantes masculinos, mientras el desempleo e inestabilidad laboral fueron vinculados por otro $17.5 \%$ al contexto de la vida masculina. El análisis temático fue dirigido por los siguientes temas: Prácticas particulares del consumo de alcohol como subyacente a la percepción de la masculinidad en el contexto etnocultural de habla portuguesa; y: Tendencias del consumo de alcohol influenciado por el origen etnocultural, presión social, y respuesta a los factores estresantes como riesgo potencial de cáncer. Conclusión- Los participantes recomendaron una mayor accesibilidad a los resultados de la investigación, demostrando la relación entre el consumo de alcohol y el riesgo incrementado de desarrollo de cáncer, como factor de desmitificación de la inocuidad del alcohol en relación al cáncer. La investigación multidisciplinaria y conjunta entre el trabajo de Enfermería y el oncológico pueden re direccionar las políticas preventivas de cáncer en los hombres. Los esfuerzos futuros deben incluir acciones tanto a nivel individual como colectivo, con medidas para actuar sobre factores de riesgo que puedan aumentar el riesgo de cáncer.

Palabras clave: Comportamientos de riesgo para la salud; Consumo de bebidas alcohólicas; Masculinidad; Neoplasias; Salud del hombre.

\section{Introdução}

Atualmente, o álcool é uma substância psicoativa consumida em diversos países do mundo, contribuindo para que ocorra uma incidência elevada de mortes e de inúmeros outros agravos à população. Seu consumo abusivo configura importante fator associado à adoção de condutas de risco, acidentes, transtornos afetivos, bem como ao desenvolvimento de câncer, de outras doenças e dependência (Barros \& Costa, 2019). Para abordar suas consequências, ênfase tem sido dada à assistência oncológica, mobilizando esforços para mudança na sistematização de registros, acompanhamento e intervenção no âmbito da vigilância (Instituto Nacional de Câncer José Alencar Gomes da Silva [INCJAGS], 2019), bem como nos modelos de atenção direcionados à população (ex.: promoção, prevenção, cura e reabilitação em saúde) (Munhoz et al., 2017). O álcool é considerado um pró-carcinogênico bem definido (Jürgen, Shield, \& Weiderpass, 2020; Rehm \& Shield, 2021; Zaitsu, Takeuchi, Kobayashi, \& Kawachi, 2020).

A Agência Internacional de Pesquisas Sobre Câncer (IARC), vinculada à Organização Mundial de Saúde (Sarich et al., 2021), compilou dados de 185 países, ratificando as expressivas diferenças na magnitude e perfil do câncer nos diferentes continentes. No contexto europeu, o consumo de álcool é fator de risco para câncer de cólon e reto (29\%), câncer de mama (nas mulheres) (15\%), esôfago (14\%) câncer de lábios e oral (12\%), faringe (excluindo nasofaringe) (12\%), fígado (12\%), e laringe (6\%) (World Health Organization, 2018). O álcool também representa risco para câncer de próstata para homens japoneses (Rehm \& Shield, 2021) e para câncer de pulmão para homens nos 37 países da Organização para Cooperação e Desenvolvimento Econômico localizados na América do Norte e do Sul, Europa e Pacífico asiático (Moryson \& StawińskaWitoszyńska, 2021). A mortalidade para câncer de pulmão em homens é alta em todos os países analisados, exceto a Islândia (Moryson \& Stawińska-Witoszyńska, 2021). Na Austrália, homens com mais de 50 anos com diagnóstico de hepatopatia de 
causa alcoólica demonstraram alto risco para câncer de fígado (Hagström et al., 2010) e homens com mais de 45 anos demonstraram tendência a consumir mais álcool semanalmente em associação ao frequente consumo de carne vermelha (Zaitsu et al., 2020).

Na população masculina, destaca-se que um em cada cinco homens desenvolverá câncer ao longo da vida e um a cada oito morrerá de câncer no mundo (Sarich et al., 2021). No Brasil, o Instituto Nacional de Câncer (INCA) estimou para o triênio 2020-2022 a incidência de aproximadamente 177 mil casos de câncer de pele tipo não melanoma e 450 mil casos de outras modalidades (INCJAGS, 2019). Os tipos de câncer mais incidentes em homens, excluindo-se os de pele não melanoma serão os de próstata (29.2,\%), cólon e reto (9.1\%), pulmão (7.9\%), estômago (5.9\%) e cavidade oral (5\%), com uma taxa ajustada por idade em torno de 215.86/100.000 mil habitantes, considerada intermediária e compatível com as apresentadas por países em desenvolvimento (INCJAGS, 2019). Destacam-se as altas taxas câncer de laringe na região da América do Sul entre os homens brasileiros, com registro de 5.9 casos novos por 100.000 homens (Costa et al., 2021).

Nas bebidas alcoólicas cuja quantidade de etanol e em teores diferenciados comumente se apresenta como principal agente agressor (Silva, 2019). Estima-se também que essa correlação e probabilidade podem ser ampliadas à medida que o consumo se associa com outros fatores, dentre os quais se destaca o tabagismo (Barbosa, Pereira, Cruz \& Leite, 2018).

Recente estatística internacional revela que entre os países com os mais elevados índices de grande consumidores de bebidas alcoólicas estão os homens (Sarich et al., 2021). O consumo elevado de álcool relaciona-se com vários efeitos adversos à saúde, tais como todas as causas de mortalidade, câncer, enfermidades cardiovasculares e traumas físicos (Jani et al., 2021). O consumo ocorre em homens maiores de 15 anos (Sarich et al., 2021), fazendo parte do elenco de comportamentos de risco amplamente documentados na literatura internacional aqueles relativos ao conceito de masculinidade hegemônica ou tradicional (Courtenay, 2011; Pinheiro et al., 2017; Zanchetta et al., 2017). O consumo de álcool pode estabelecer nexos concretos com crenças e influências culturais de um país e/ou de grupos populacionais, desempenhando diversas funções e significados no mundo (Sarich et al., 2021). O consumo excessivo faz parte da cultura jovem masculina (Sarich et al., 2021) ocasionando impactos à saúde e outros desdobramentos sociais (Munhoz et al., 2017).

Esta pesquisa concentrou-se neste problema global de saúde masculina na cultura latino-americana. A definição da cultura latino-americana tem suas raízes na colonização européia iniciada no século 16 e inclui sua identidade linguística pelos idiomas Espanhol, Francês e Português. Ainda outras origens latinas ligam-se à cultura italiana como um dos berços culturais. Assim, as origens têm profundos laços e conexões entre a América Latina e a Europa e essas, com o mundo, fato historicamente constatado e ainda muito mais evidente nos dias atuais (Finn \& Hanson, 2017). A cultura latino-americana identifica também 21 países localizados nas Américas do Sul e do Norte. Cumpre destacar que informações somente sobre o idioma de uso diário constitui um fator de identificação menos apropriado para a identificação de grupos étnicos, mas podem ser usadas para se entender a assimilação linguística em grupos de imigrantes. Entretanto, informações sobre o país de nascimento devem ser correspondentes (United Nations, n. d.).

Nesse transcontinental grupo étnico-cultural foi documentada a alta prevalência de episódios de consumo elevado (definida como o consumo de 60 gramas ou mais de puro álcool ao menos em uma única ocasião mensal (Jürgen et al., 2020)) entre os homens maiores de 15 anos. Sendo: França (68.6\%), Perú (68.6\%), Brasil (66.7\%), Portugal (65.4\%) e Itália (57.6\%)(Sarich et al., 2021). Cumpre destacar que os padrões de consumo de álcool e comportamentos dos brasileiros em relação a bebidas alcoólicas por homens referem-se a um início em torno de 18 anos,com 5 doses em eventos sociais. Ademais, resultados relativos a outros $421(30.4 \%)$ homens brasileiros que associaram o consumo abusivo de álcool a comportamentos de risco à saúde (Arruda \& Marcin, 2018; Oliveira, Azambuza, \& Santos, 2015).

Neste artigo apresentamos resultados parciais de uma pesquisa internacional multilíngue com homens de cultura latina, entre os quais encontram-se os homens lusófonos. Como a maior amostragem da pesquisa foi composta por tais 
respondentes, este artigo destaca os resultados desse segmento, sem que haja nenhuma outra justificativa para preferência ou escolha da amostragem. Diante do exposto, as perguntas de pesquisa originais que guiaram a pesquisa online e adaptadas aos homens lusofónos são as seguintes: "Como a cultura etnocultural lusófona molda as visões sobre masculinidade e os comportamentos de consumo alcoólico?" e, "Como os homens lusófonos percebem o uso multifacetado do álcool como situações potencialmente de risco para câncer entre os homens?”.

Os objetivos gerais originais que nortearam a pesquisa também adaptados aoss homens lusófonos foram: Identificar as práticas do consumo alcoólico segundo a percepção de masculinidade no contexto etnocultural lusófono; e Analisar as percepções de homens lusófonos, de origem latina, acerca do uso multifacetado do álcool como situações potencialmente de risco para o desenvolvimento de câncer.

Esta pesquisa inspirou-se no modelo canadense de promoção da saúde populacional (Government of Canada, 2001) que incorpora os determinantes sociais de saúde (ex.: redes de apoio social, práticas de saúde pessoais, sexo, etc.) como dimensões da promoção da saúde que tratam das condições de vida, isto é, aquelas relacionadas aos lugares e circunstâncias nas quais os indivíduos nascem, vivem, trabalham e se desenvolvem (Canadian Council of Social Determinants of Health, 2015). Este modelo reconhece a implementação de ações extensivas de promoção da saúde, tais como, o fortalecimento da ação comunitária, a criação de ambiente de apoio, o desenvolvimento de habilidades pessoais, a criação de políticas públicas saudáveis e a reorientação dos sistemas de saúde para maximizar os determinantes sociais da saúde com intervenções aos níveis individuais, familiares e da sociedade.

\section{Metodologia}

Pesquisa descritiva exploratória, de abordagem quanti-qualitativa (Grove, Burns, \& Gray, 2013), utilizando a modalidade de sondagem online para a coleta de dados. A pesquisa reportada trata-se de um recorte de uma pesquisa abrangendo respondentes de origem etnocultural latina. Dentro da perspectiva cultural, encontra-se a língua materna, que confirma a identidade etnocultural latina. A pesquisa teve como alvo homens integrantes desta origem e que se comunicavam nos idiomas português, espanhol, francês, inglês (principalmente os imigrantes de segunda e terceira geração que vivem na América do Norte) ou italiano. Este artigo reporta apenas os dados dos homens maiores de 18 anos, que se auto identificaram como sendo de origem latina e que responderam o questionário online em português.

Foi utilizado um questionário original (criado pela primeira e quarta autoras) composto de oito questões, sendo três de identificação sociodemográfica, três perguntas fechadas e duas perguntas abertas. O questionário explorou as variáveis idade, país de residência e grupo de identificação cultural. As perguntas fechadas exploraram, pela seleção de afirmações, as percepções dos respondentes quanto: ao uso social do álcool e aos pensamentos comuns no país de residência sobre consumo de álcool; às áreas profissionais nas quais os homens estariam propensos a beber mais e, por isso, os colocariam em risco mais elevado para câncer; e às condições de vida profissional e pessoal nas quais o homem usa o álcool para lidar com situações específicas, colocando-os em risco mais elevado para o desenvolvimento de câncer. As perguntas abertas exploraram o quanto o consumo de bebida alcoólica era considerado uma prática comum e aceitável para homens de todas as idades; e sobre o que os respondentes pensavam acerca da maior probabilidade de incidência de câncer entre pessoas consumidoras de bebidas com maior teor alcoólico.

O recrutamento se deu por meio da publicação de anúncios contendo o link para consentimento informado e acesso às questões da pesquisa nas páginas do Facebook dos membros da equipe de pesquisa, estimulando seus contatos online a reencaminhá-lo para suas redes sociais. A equipe ainda enviou os anúncios por e-mail aos indivíduos de suas redes profissionais e sociais para alcançar os mais distantes possíveis participantes. Além disso, os pesquisadores que são professores universitários fizeram contato com associações de estudantes em seus respectivos campi para colaboração na publicação do 
anúncio da pesquisa entre os grupos de estudantes do sexo masculino. Devido à adesão espontânea dos respondentes em resposta ao convite direto ou indireto dos pesquisadores e de colaboradores na divulgação da pesquisa, o método de seleção foi o não probabilístico combinado com o método bola de neve, compatíveis também com seu uso na sondagem online (Sue \& Ritter, 2016).

Como critérios de inclusão aplicável a este recorte lusófono, considerou-se: ter no mínimo 18 anos de idade; ter ascendência familiar portuguesa ou brasileira; se auto identificar como homem; e decidir participar voluntariamente desta pesquisa respondendo ao questionário online. Os critérios de exclusão foram: ter menos de 18 anos; não se identificar como tendo herança étnico-cultural portuguesa ou brasileira; não se auto identificar como homem, e não pretender participar da pesquisa voluntariamente.

A coleta de dados foi realizada pela aplicação de um questionário online disponibilizado em Português na plataforma Opinio, da Universidade Ryerson (Toronto-Canadá). O período de coleta de dados ocorreu entre maio de 2018 e março de 2019.

Os dados sociodemográficos foram compilados com estatística descritiva (Grove et al., 2013). O texto das respostas abertas foi submetido à leitura crítica e, em seguida, submetido a procedimentos inspirados na técnica de análise temática (Paillé \& Mucchielli, 2016):

- leitura intensiva das transcrições com anotações das ideias emergentes;

- preparação de uma lista preliminar de temas para guiar a leitura subsequente das transcrições. Nesta pesquisa, os temas preliminares foram: Práticas particulares do consumo alcoólico segundo a percepção de masculinidade no contexto etnocultural brasileiro; e, Tendências do consumo alcoólico influenciado pela origem etnocultural, pressão de colegas, e resposta a fatores estressantes no cotidiano como risco potencial para o câncer;

- identificação de conceitos analíticos que destacam afinidades e contradições entre os temas e,

- registro das reflexões provocadas durante a leitura das transcrições agrupando os temas, refletindo sobre os temas centrais, para responder às perguntas de pesquisa.

Esta pesquisa foi aprovada pelo Comitê de Ética em Pesquisa da Universidade Ryerson (REB 2018-119) (TorontoCanadá), cumprindo todas as exigências éticas estabelecidas para estudos envolvendo seres humanos. O consentimento livre e informado foi obtido com base nos princípios do consentimento implícito. Entende-se por consentimento implícito a ação do participante de acessar a plataforma online Opinio e clicar (avançar) no link fornecido nos anúncios, indicando que o consentimento foi concedido pelo participante, embora não tenha sido expresso verbalmente ou por escrito.

\section{Resultados e Discussão}

Os resultados referem-se àqueles 184 homens que se autodeclararam lusófonos e que responderam completamente o questionário. Os dados sociodemográficos apresentam predominância da faixa etária de 50 anos ou mais (21.7\%), permitindo identificar que a maioria dos respondentes moram no Brasil (89\%), tendo sua origem cultural brasileira (70.1\%) (Tabela1). 
Tabela 1 - Dados Sociodemográficos dos Respondentes.

\begin{tabular}{lcc}
\hline Dados sociodemográficos & Frequência absoluta (n) & Frequência relativa \\
\hline Faixa etária & $\mathbf{n}$ & $\%$ \\
$19-29$ anos & 32 & $17.4 \%$ \\
30 - 39 anos & 37 & $20.1 \%$ \\
40 - 49 anos & 28 & $15.2 \%$ \\
50 anos ou mais & 40 & $21.7 \%$ \\
Não responderam & 47 & $25.6 \%$ \\
Total & 184 & $100.0 \%$ \\
País de residência & $\mathbf{n}$ & $\%$ \\
Brasil & 164 & $89.0 \%$ \\
Canadá & 12 & $6.7 \%$ \\
Portugal & 4 & $2.2 \%$ \\
Estados Unidos & 1 & $0.5 \%$ \\
Não responderam & 3 & $1.6 \%$ \\
Total & 184 & $100.0 \%$ \\
Identidade cultural & $\mathbf{n}$ & $\%$ \\
autodenominada & 129 & $70.1 \%$ \\
Brasileiro & 1 & $0.5 \%$ \\
Português & 7 & $3.8 \%$ \\
Italiano & 47 & $25.6 \%$ \\
Não responderam & 184 & $100.0 \%$ \\
Total & &
\end{tabular}

Fonte: Dados da pesquisa.

Os resultados demonstram que o consumo do álcool integra a identidade cultural masculina, sobremaneira a de homens brasileiros. Os pensamentos mais comuns relacionados ao consumo de álcool para a grande parte dos respondentes são de que o álcool faz parte da vida dos estudantes (17.5\%) e que seu consumo é permitido e estimulado em reuniões sociais $(16.8 \%)$. Também existe uma percepção que justifica o consumo de álcool como estratégia que facilita a aceitação social e que proporciona alívio de estresse (Tabela 2). Além disso, destaca-se que construção civil (23.2\%), fabricação e produção (14.8\%) são as áreas profissionais que mais consomem álcool (Tabela 2). A total of 47 respondentes (7.1\%) não responderam sobre os pensamentos comuns nem 52 dentre eles $(\mathrm{n}=11 \%)$ quanto as areas profissionais de maior consumo 
Tabela 2 - Expressões Socioculturais do Consumo de Álcool na Perspectiva da Masculinidade.

\begin{tabular}{lcc}
\hline Pensamentos comuns sobre o consumo alcoólico & $\mathbf{n}$ & $\mathbf{\%}$ \\
\hline Álcool faz parte da vida da maioria dos estudantes masculinos & 116 & $17.5 \%$ \\
O uso de álcool não é reprimido nas reuniões sociais & 111 & $16.8 \%$ \\
O uso de álcool é uma boa maneira de se sentir aceito em grupos sociais & 102 & $15.4 \%$ \\
O uso de álcool é uma forma útil de lidar com situações estressantes & 93 & $14.1 \%$ \\
Não é incomum tomar uma bebida alcoólica quando chega em casa após o & 79 & $12.0 \%$ \\
trabalho & & \\
É comum incentivar meninos a beberem álcool em reuniões familiares em & 62 & $9.4 \%$ \\
idades mais jovens & 32 & $4.8 \%$ \\
Eu não bebo álcool & 19 & $2.9 \%$ \\
O uso de álcool é uma forma saudável de ajudar os homens a dormir & 661 & $100.0 \%$ \\
Total & $\mathbf{n}$ & $\mathbf{\%}$ \\
Áreas profissionais de maior consumo alcoólico & 108 & $23.2 \%$ \\
Construção civil & 69 & $14.8 \%$ \\
Produção/Fabricação & 56 & $12.0 \%$ \\
Policiais e Bombeiros & 51 & $11.0 \%$ \\
Profissionais de saúde & 46 & $9.9 \%$ \\
Política & 37 & $8.0 \%$ \\
Vendas & 25 & $5.4 \%$ \\
Direito & 22 & $4.7 \%$ \\
Outros & 465 & $100.0 \%$ \\
Total & &
\end{tabular}

Fonte: Dados da pesquisa.

Vale ressaltar que na opção "outros" foram listadas áreas profissionais específicas, tais como: rodoviários, estudantes de graduação, técnicos em necrotério, agricultores, auxiliares administrativos, marítimos e pescadores, professores de forma geral, garçons, profissionais de tecnologia e informática, profissionais em casas noturnas, profissional do sexo, mecânico e pintor.

Em relação às situações em que o álcool é consumido para ajudar a enfrentar situações difíceis, houve predominância de desemprego/instabilidade no trabalho (17.5\%), estresse (17.3\%) e problemas financeiros (17.0\%) (Tabela 3). Informação sobre o consumo alcoólico não foi provida por 50 respondentes $(7.7 \%)$. 
Tabela 3 - Consumo Alcoólico Relacionado ao Contexto de Vida Masculina.

\begin{tabular}{lcc}
\hline $\begin{array}{l}\text { Situações específicas que o consumo de álcool ajuda a } \\
\text { enfrentar }\end{array}$ & $\mathbf{n}$ & $\mathbf{\%}$ \\
\hline Desemprego ou instabilidade no trabalho & 114 & $17.5 \%$ \\
Estresse & 113 & $17.3 \%$ \\
Problemas financeiros & 111 & $17.0 \%$ \\
Divórcio ou separação & 106 & $16.2 \%$ \\
Morte de um ente querido & 55 & $8.4 \%$ \\
Doença ou deficiência & 38 & $5.8 \%$ \\
Problemas mentais ou psicológicos & 36 & $5.5 \%$ \\
Dor crônica & 21 & $3.2 \%$ \\
Outros & 9 & $1.4 \%$ \\
Total & $\mathbf{6 5 3}$ & $\mathbf{1 0 0 \%}$ \\
\hline
\end{tabular}

Fonte: Dados da pesquisa.

Os respondentes ainda mencionaram outras situações e condições diversas, tais como compulsão, solidão, reunião social, prazer pelo efeito do álcool e eventos esportivos/artísticos relacionados ao consumo contextualizado pelos homens.

Sobre o questionamento acerca de o "quanto consumir bebida alcoólica é considerada uma prática comum e aceitável para homens de todas as idades", 141 (76.6\%) respondentes compartilharam suas opiniões e conhecimentos relacionados à presença do álcool nas atividades familiares e sociais, ambas marcadas por uma certa banalização do consumo alcoólico entre os homens. Segundo os respondentes, a prática social e familiar amplamente aceitável, e quase que imposta, encontra-se enraizada em normas culturais e age como confirmação social de maturidade, masculinidade e virilidade: a cultura machista incentiva o uso de bebidas alcoólicas ligando a imagem do homem que aproveita a vida, paquera as mulheres, se distrai com amigos. Como relatado por um homem brasileiro: Também, é comum vermos pais incentivando seus filhos homens a beber, desde passar a chupeta do menino na cerveja a permitir goles pequenos a crianças.

Segundo os respondentes, no macrocontexto social brasileiro, o estímulo frequente ao consumo alcoólico leva a uma percepção de naturalidade e inocuidade: $\mathrm{O}$ consumo de bebida alcoólica por homens é extremamente estimulado desde a infância e... na prática, as bebidas alcoólicas são de fácil acesso aos adolescentes.

Segundo ainda os respondentes, sob a égide da força cultural do conceito de masculinidade tradicional ou hegemônica, o consumo de álcool consolida os traços característicos do universo masculino, criado e imaginado no Brasil e em Portugal. Como relatado por um homem português: Em Portugal, é quase que uma obrigação ou imposição de masculinidade beber álcool entre os homens.

A segunda pergunta concentrou-se em evidências científicas sobre a relação entre consumo de bebidas de alto teor alcoólico e risco para o desenvolvimento de câncer: Pesquisas relatam que pessoas que consomem bebidas com 40-43\% de teor alcoólico (ex. vodka, uísque, rum e outros tipos) têm risco mais elevado para o câncer. O que você pensa sobre isso?

A simples leitura das respostas claramente indicava os pensamentos dos homens com frases iniciadas por: Acredito sim...; Não sabia...; Não acredito..., Não sei opinar...; e permite identificar que existe uma tendência de pensamento que os homens têm sobre o resultado de pesquisas sobre o risco para o desenvolvimento de câncer. As narrativas oferecidas por 63 homens (49.21\%) dentre os 128 que responderam esta pergunta indicaram que eles acreditavam no resultado sobre tal risco, como comentado: Faz sentido. Se o etanol é que provoca o risco para câncer, quanto maior o teor alcoólico de uma bebida, maior será o risco para câncer.

Por outro lado, apenas 12 homens (9.37\%) informaram não saber de tal risco: Nunca tinha ouvido a relação até então, e, sem saber quais tipos de câncer, não consigo imaginar os motivos. 
Outro achado digno de observação refere-se a 13 homens (10.15\%) que sugeriram que tal evidência científica deva ser divulgada ao grande público como uma necessidade de maior democratização de tal tipo de informação científica: Que esse alerta deveria ser feito de forma bem clara e direta como nos maços de cigarro.

Apenas 6 homens $(4.68 \%)$ consideraram a possibilidade de tal risco com alguma reflexão: É possível, pois os componentes das bebidas alcoólicas, na maioria das vezes, são desconhecidos, o consumo, o tipo, a frequência, o tempo de uso e o trajeto que a bebida alcoólica passa pelo corpo humano pode gerar vários tipos de cânceres.

Dentre os 23 homens (17.96\%) que não responderam diretamente à pergunta, alguns explicaram que o risco depende do volume consumido, ou da combinação entre álcool e tabaco. Eles emitiram opiniões diversas sobre aspectos paralelos, subjacentes e complementares a simples questão de consumir ou não bebidas de alto teor alcoólico e a pesquisa demonstrando risco elevado para o desenvolvimento de câncer. Enquanto poucos indicavam dúvidas e credibilidade parcial dos achados científicos, a maioria dos respondentes demonstrou, em termos gerais, que a pergunta os estimulou a refletir sobre o tema:.. percebo ainda que as bebidas de "baladas" estão em alta entre os jovens estudantes, são bebidas como vodka, misturadas a estimulantes, e ainda o consumo "casado" que é bebidas e drogas, como o crack.

Apesar de as pesquisas associarem o consumo de bebidas alcoólicas como fator de risco para o desenvolvimento de câncer e de este consumo ser altamente influenciado culturalmente na população masculina, os respondentes conceitos e entendimentos sobre o álcool, seu consumo e suas consequências em concordância com as pesquisas divulgadas sobre o tema.

\section{Análise temática}

A sociedade lusófona tende a banalizar o consumo do álcool pelos homens como uma prática influenciada pelo sentimento de invisibilidade ligada aos valores patriarcais. Para os homens, o álcool está relacionado a construções culturais de masculinidade, vinculado à identidade e à autoimagem moldadas e sua aceitação geral permeada em grupos sociais, mas também dentro da própria família, muitas vezes, começando na mais tenra idade. Frente aos resultados encontrados, são apresentadas duas dimensões temáticas:

\section{Práticas particulares do consumo alcoólico segundo a percepção de masculinidade no contexto etnocultural lusófono.}

Entre a banalização do álcool e a glamourização de seu consumo principalmente no Brasil, o consumo de bebidas alcoólicas nas diferentes faixas etárias caracteriza-se como hábito inerente e norteador de comportamentos e ações, cujas consequências físicas e psicossociais são extremamente danosas entre jovens e adultos. Todavia, ainda é insipiente o arsenal teórico abrangendo o consumo do álcool entre determinados grupos populacionais, como o dos idosos (Barbosa et al., 2018). Devido à ampla divulgação na mídia, o consumo de bebidas com alto teor alcoólico, e os problemas que dele decorrem, estão se tornando cada vez mais acessíveis à população geral (Evangelista, Kadooka, \& Pires, 2018). Favorecendo a sociabilidade e a interação entre pessoas, especialmente aqueles em condições de vulnerabilidade emocional devido à ansiedade, estresse e baixa autoestima (Evangelista et al., 2018), o álcool se imbrica na expressão da masculinidade hegemônica (Courtenay, 2011; Pinheiro et al., 2017; Zanchetta et al., 2017) em experiências de doenças sob a ótica dos determinantes sociais da saúde (Zanchetta, Kaszap, Monteiro, Gorospe IV, \& Pilon, 2011).

O consumo de álcool é uma prática muito comum entre os homens. Eu não diria que é só algo "aceitável", mas é uma prática vista no meio masculino como símbolo de virilidade.

Imprudente seria interpretar o consumo de álcool como uma conduta natural e inquestionável (Zanchetta et al., 2018), inclusive entre os homens de qualquer idade. O conhecimento dos fatores de risco, dentre eles o sexo biológico e a construção cultural da masculinidade e seus modos de viver, pode influenciar o estabelecimento de medidas profiláticas que podem repercutir de maneira positiva (ou não), diminuindo a possibilidade de os indivíduos expostos adoecerem (Silva, 2019). 
Tendências do consumo alcoólico influenciado pela origem etnocultural, pressão social, e resposta aos fatores estressantes no cotidiano como risco potencial para o câncer.

Os resultados corroboram que, para os homens, o álcool representa recurso utilizado para fuga do estresse, sendo utilizado como elemento que facilita/contribui para a sua aceitação social, e, por ser uma mercadoria de baixo custo financeiro, seu consumo se consolidou entre profissões estressantes e como recurso utilizado para enfrentamento de momentos de dificuldade na vida. Tais tendências foram confirmadas pelas falas dos respondentes e se revelam por meio de justificativas que respaldam seu consumo, variando desde a ausência de repressão a até mesmo como estratégia para possibilitar maior interação social e capacidade de enfrentamento de situações estressantes.

Os determinantes socioeconômicos, de maneira simultânea, também são capazes de ampla e negativamente influenciar a população, submetendo-a a níveis variados de exposição a agentes carcinogênicos, tal como o álcool (Lima \& Dimenstein, 2018). Cultura é outro determinante social da saúde que, sob a influência daqueles socioeconômicos, pode redesenhar possíveis mudanças em valores e crenças sobre o consumo alcoólico e riscos para a saúde.

Vejo que bebidas com esse teor alcoólico são ... por pessoas que trabalham na construção civil e atividades com maior desgaste físico. E essas bebidas são de baixo custo aqui, como cachaça. Bebidas como uísque, com processos de destilação mais apurado, são consumidas aqui no Brasil por empresários, advogados e pessoas com mais recursos etc., porque custam bem mais caro aqui. As bebidas com teor alcoólico menor (cervejas e chope) são as mais consumidas no Brasil.

Outra importante influência de determinantes sociais refere-se à educação e a práticas saudáveis de saúde e habilidades de enfrentamento do estresse, pois os respondentes acreditam que o consumo de álcool entre profissionais de saúde seja frequente. A percepção de tal tendência pode indicar que tais profissionais tenham conhecimento científico, entretanto, ainda que seja insuficiente, não seja utilizado adequadamente por eles, no sentido de prevenção do câncer e cuidado com sua própria saúde (Felipe \& Gomes, 2014; Kfouri et al., 2018; Machado, Monteiro, Ribeiro, \& Guilhem, 2016).

Apesar das pesquisas abrangentes sobre os riscos associados às bebidas alcoólicas, o conhecimento do público em geral sobre álcool permanece difícil de se definir.

Pesquisas têm vieses e devem ser analisadas como um todo. É uma correlação ou causalidade? O grupo teste foi acompanhado durante quanto tempo? Ela ainda está em andamento ou foi concluída? Acima de tudo, é preciso entender que não existe consumo seguro sem moderação.

Entre homens que se autodeclararam consumidores leves a moderados, o risco de câncer relacionado ao álcool foi considerado como inevitável, mesmo considerando ser inútil a mudança de comportamento, assim como a ideia de que o consumo de álcool é parte normal e necessária da vida.

Analisando crítica e objetivamente os resultados, pode-se inferir que a forma como a produção científica existente está sendo socializada e utilizada pela população ainda não se mostra suficientemente eficaz para impactar e gerar as mudanças necessárias no contexto em destaque. Existem, portanto, urgência e necessidade de implementação de estratégias de divulgação para que esse conhecimento circule de maneira acessível, com linguagem clara e de forma mais abrangente, fazendo maior sentido para a sociedade, de modo a suscitar transformações eficazes em seus padrões de consumo de álcool em longo prazo e, por consequência, resultar na minimização dos impactos para risco de câncer. Tal ação dever ser realizada também entre as famílias, no ambiente de trabalho, nas escolas de ensino médio, etc. 


\section{Discussão}

Os resultados evidenciaram que os homens lusófonos consideram que o consumo de álcool estabelece relações com fatores de ordem diversa, ocorrendo sob forte influência psicossocial em associação com questões políticas, socioeconômicas, socioculturais, regras de conduta e, sobremaneira psicossociais.

Considerando que a maior parte dos respondentes reside no Brasil e/ou refere identidade cultural brasileira, país onde o álcool é uma droga lícita, de certo modo com pequeno custo e de fácil acesso à população, esses fatores colaboram para que muitas pessoas iniciem seu consumo ainda jovens (Junqueira et al., 2017), perpetuando-se ao longo da sua vida.

A associação do câncer de cabeça e pescoço com o consumo de álcool e tabaco foi identificada em um estudo realizado em 3 diferentes regiões do Brasil, apresentando a fração atribuível ao consumo de bebidas alcoólicas mais alta no Sudeste (78\%) e Sul (77\%) do que no Centro-Oeste (62\%), o que condiz com outros levantamentos nacionais que identificam o maior consumo de álcool nas regiões Sudeste e Sul do país (Lima \& Dimenstein, 2018).

É válido refletir acerca das principais atividades laborais mencionadas pelos respondentes como de maior risco, sendo as desempenhadas em áreas conhecidamente mal remuneradas e que os expõem a riscos ocupacionais elevados, como também questões relacionadas à instabilidade econômica que vigora no país. Acrescido a isso está o consumo, muitas vezes excessivo, servindo como alternativa para a legitimação de condutas e suas interações sociais, para amenizar o sofrimento psíquico e para a fuga momentânea da realidade. O uso prazeroso do álcool induz à repetição (Silva, 2019), e como o estresse, o sofrimento psíquico e o uso de psicoativos aumentaram significativamente nos últimos anos (Junqueira et al., 2017), influenciando, de maneira negativa, o consumo abusivo de bebidas alcoólicas associado a qualquer um desses fatores entre os homens, em geral, pode-se induzir a uma futura dependência.

Na perspectiva do autocuidado, quanto menor a autopercepção acerca da saúde, maior é a possibilidade de exposição a esse fator de risco (Monteiro et al., 2018). Considerando as respostas fornecidas pelos respondentes, nas quais se observa que boa parte tem conhecimento dessa condição e não a valoriza, a ponto de suprimir o consumo, e que uma outra parcela não detém esse mesmo conhecimento, essa é uma realidade preocupante e que não deve ser negligenciada.

Partindo do entendimento de que comportamento em saúde é qualquer hábito socialmente construído, que tem a capacidade de impactar positiva ou negativamente na saúde de uma pessoa (Arruda \& Marcon, 2018), o consumo de bebidas alcoólicas é um comportamento que pode ser modificado. Deve-se envidar esforços para superar as dificuldades verificadas nos serviços públicos para alcançar os homens e trazê-los à cena, não apenas como receptores de informações e cuidados desvinculados de suas reais necessidades, mas, sobretudo, enquanto protagonistas da construção de sua condição de saúde.

Esforços para reduzir o impacto do câncer devido ao consumo de álcool devem incluir ações individuais e coletivas, bem como medidas para atuar sobre os fatores de risco que podem aumentar o risco de câncer (Jürgen et al., 2020). A atenção deve ser redobrada para homens com perfil socioeconômico caracterizado por privações, já que esses tendem a ter associados riscos ao tabagismo (Jani et al., 2021). Recentes esforços concentram-se em intervenções educativas para a prevenção do câncer de cabeça e pescoço e outros cânceres, inclusive com a população jovem (Marziliano, Teckie, \& Diefenbach, 2020). Observa-se algum sucesso para se manter comportamentos preventivos com o uso de dispositivos eletrônicos (aplicativos para smartphones e mensagens de texto) (Marziliano et al., 2020). Entre os múltiplos fatores a serem considerados, está a inegável influência econômica da indústria de bebidas alcoólicas presente nos acordos comerciais, na legislação, nas relações com os governos, assim como com a mídia profissional, tal como alertado por autores australianos (Miller et al., 2021). Esses autores destacam que há distorção da mensagem publicitária sobre os benefícios do álcool para a saúde, principalmente pela indústria do vinho. Outra abordagem australiana revela a minimização de danos a curto prazo por meio de campanha de educação de massa pela mídia, maior regulamentação da indústria, com responsabilização legal quanto aos serviços de venda e consumo de álcool, leis de teste de respiração (bafômetro) aleatório, com especial foco na população jovem (Zaitsu et al., 2020). 


\section{Conclusão}

Esta pesquisa contribui empiricamente para o aprofundamento sobre o assunto permitindo consubstanciar argumentos necessários à sua compreensão, no âmbito do ensino e da pesquisa, viabilizando novas formas de pensar e de cuidar. E, ainda, contribuir na ampliação da conscientização acerca dos riscos que o consumo de álcool acarreta para o desenvolvimento de câncer e a adoção de medidas preventivas mais coerentes e adequadas. Por exemplo, o departamento de comunicação social de empresas, universidades, organizações profissionais, associações de classe de profissionais da saúde (Enfermagem, Medicina, Psicologia, Serviço Social, Terapia Ocupacional, etc) deveriam adotar campanhas de esclarecimento sobre o consumo do álcool como estratégia para lidar com frustrações e estresses, seus consequentes riscos à integridade física e os efeitos cumulativos, com risco elevado para o desenvolvimento de neoplasias. Ações específicas aos homens deveriam se concentrar no ensino de estratégias saudáveis e responsivas às questões da masculinidade em suas múltiplas formas, tal como vivenciado nas sociedades lusófonas sobre lidar com situações emocionais sem o recurso do consumo de álcool. A prevenção deveria assim considerar as áreas de ações intersetoriais integradas como propostas no modelo canadense de promoção da saúde populacional.

Cumpre destacar, que tal modelo vem sendo aplicado em vários países desde a assinatura da Carta da Promoção da Saúde de Ottawa (Ministério da Saúde, n. d.), entre eles, o Brasil, principalmente em razão da cooperação científica estabelecida com o Canadá (Ministério das Relações Exteriores, 2010). No Brasil, cabe destacar que existe uma tendência para a aplicação parcial do modelo devido à tradicional ênfase na ação educativa para a clientela, ou seja, no desenvolvimento de habilidades individuais sem que as outras quatro de ações integradas sejam contempladas. Quando este modelo é aplicado para a promoção da saúde - para conscientização sobre riscos e alternativas para coibir o consumo do álcool de modo a prevenir o câncer na população masculina -, deveria haver colaboração entre a mídia profissional e as mídias sociais, a indústria de produtores de bebidas alcoólicas, seguradoras de acidentes, Ministério do Trabalho, Secretaria da Previdência, seguros privados de saúde e representantes da sociedade civil, todos advogando sobre a prevenção de mortes decorrentes de acidentes automobilísticos, violência familiar, violência comunitária etc.

Importante destacar que uma das limitações da pesquisa encontra-se em sua restrita representatividade de homens lusófonos que vivem em muitos outros países, devido ao alcance limitado do recrutamento na comunidade global lusófona transcontinental. Portanto, a transferibilidade da evidência encontra-se limitada aos países reportados pelos respondentes.

\section{Agradecimentos}

Os autores agradecem ao Enfermeiro Ricardo Espíndola pela tradução do resumo para o idioma espanhol, e a Sra.

Sandra Andrade do Val pela revisão técnica gramatical do manuscrito no idioma português.

\section{Referências}

Arruda, G. O., \& Marcon, S. S. (2018). Comportamentos de Risco à Saúde de Homens da Região Sul do Brasil. Texto Contexto Enferm, $27(2)$, e2640014. https://doi.org/10.1590/0104-070720180002640014

Barbosa, M. B., Pereira, C. V., Cruz, D. T., \& Leite, I. C. G. (2018). Prevalência e Fatores Associados ao Consumo de Álcool e de Tabaco em Idosos Não Institucionalizados. Rev. Bras. Geriatr. Gerontol, 21(2), 125-135. https://doi.org/10.1590/1981-22562018021.170185

Barros, M. S. M. R., \& Costa, L. S. (2019). Perfil do Consumo de Álcool entre Estudantes Universitários. Rev. Eletrônica Saúde Mental Álcool Drog, 15(1), 4-13. https://10.11606/issn.1806-6976.smad.2019.000353

Canadian Council on Social Determinants of Health. (2015). A Review of Frameworks on the Determinants of Health. http://ccsdh.ca/images/uploads/Frameworks_Report_English.pdf

Costa, S. N. L., Fernandes, F. C. G. M., Souza, D. L. B, Santos, E. G. O., Bezerra, H. S., \& Barbosa, I. R. (2021). Incidence and Mortality by Larynx Cancer in Central and South America. Rev Gaúcha Enferm, 42, e20190469. https://doi.org/10.1590/1983- 1447.2021.20190469 
Courtenay, W. (2011). Dying to Be Men: Psychosocial, Environmental and Biobehavioral Directions in Promoting the Health of Men and Boys. New York: Routledge.

Evangelista, V. M. A., Kadooka, A., Pires, M. L. N., \& Constatino, E. P. (2018). Padrões e Consumo de Álcool entre Estudantes Universitários. Rev Psi Divers Saude, 7(2), 192-204. https://doi.org/10.1590/S0102-311X2011000800016

Felipe, I. C. V., \& Gomes, A. M. T. (2014). Alcohol Consumption among Academics in the Health Area: Implications for Professional Practice. Rev. Enferm. UERJ, 22(1), 35-42. http://www.facenf.uerj.br/v22n1/v22n1a06.pdf

Finn, J. C., \& Hanson, A.-M. (2017). Critical Geographies in Latin America. JLAG, 16(1), 1-15. http://www.jstor.org/stable/44861309.

Grove, S., Burns, N., \& Gray, J. R. (2013). The Practice of Nursing Research: Appraisal, Synthesis and Generation of Evidence. 7 ed. St. Louis, MI: Elsevier Saunders.

Government of Canada. (2001). Population Health Promotion: An Integrated Model of Population Health and Health Promotion. https://www.canada.ca/en/public-health/services/health-promotion/population-health/population-health-promotion-integrated-model-population-health-healthpromotion/developing-population-health-promotion-model.html

Hagström, H., Thiele, M., Sharma, R., Simon, T. G., Roelstraete, B., Söderling, J., \& Ludvigssen, J. F. (2021). Risk of Cancer in Biopsy-proven Alcoholrelated Liver

Disease: A Population-based Cohort Study of 3,410 Persons. Clin.Gastroenterol. Hepatol. (no prelo). https://doi.org/10.1016/j.cgh.2021.01.005

Instituto Nacional de Câncer José Alencar Gomes da Silva. (2019). Estimativa 2020: Incidência de Câncer no Brasil. INCA. https://www.inca.gov.br/sites/ufu.sti.inca.local/files//media/document//estimativa-2020-incidencia-de-cancer-no-brasil.pdf

Jani, B. D., McQueenie, R., Nicholl, B. I., Field, R., Hanlon. P., Gallacher, K. I...Lewsey, J. (2021). Association between Patterns of Alcohol Consumption (beverage type, frequency and consumption with food) and Risk of Adverse Health Outcomes: A Prospective Cohort Study. BMC Med, 19, 8. https://doi.org/10.1186/s12916-020-01878-2

Junqueira, M. A. B., Ferreira, M. C. M., Soares, G. T., Brito, I. E., Pires, P. L. S., Santos, M. A., ...Pillon, S. C. (2017). Uso de Álcool e Comportamento de Saúde entre Profissionais da Enfermagem. Rev. Esc. Enferm. USP, 51, e03265. http://dx.doi.org/10.1590/s1980-220x2016046103265

Jürgen, R., Shield, K. D., \& Weiderpass E. (2020). Alcohol Consumption. A Leading Risk Factor for Cancer. Chem. Biol. Interact, 331, 109280. https://doi.org/10.1016/j.cbi.2020.109280

Kfouri, S. A., Neto, J. E., Koifman, S., Curado, M. P., Menezes, A., Daudt, A. W., \& Filho, V. W. (2018). Fração de Câncer de Cabeça e Pescoço Atribuível ao Tabaco e ao Álcool em Cidades de Três Regiões Brasileiras. Rev Bras Epidemiol, 21, e180005. https://10.1590/1980-549720180005

Lima, A. I. O., \& Dimenstein, M. (2018). O consumo de Álcool e Outras Drogas na Atenção Primária. Cad Brasileiros de Saude Mental, 10(26), 46-65. https://periodicos.ufsc.br/index.php/cbsm/article/view/69044/41528

Machado, A. S., Monteiro, P. S., Ribeiro, L. M., \& Guilhem, D. (2020). Consumo de Álcool por Enfermeiros e Implicações para o Trabalho em Saúde Revisão Integrativa. Cogitare Enferm, 21(4), 1-8. http://docs.bvsalud.org/biblioref/2017/07/846694/45976-190900-1-pb.pdf

Marziliano, A., Teckie, S., \& Diefenbach, M. A. (2020). Alcohol-related Head and Neck Cancer: Summary of the Literature. Head \& Neck, 42, 732-738. https://doi.org/10.1002/hed.26023

Miller, M., Wilkinson, C., Room, R., O'Brien, P., Townsend, B., Schram, A., \& Gleeson, D. (2021). Industry Submissions on Alcohol in the Context of Australia's Trade and Investment Agreements: A Content and Thematic Analysis of Publicly Available Documents. Drug Alcohol Rev, 40, 22-30. $10.1111 /$ dar. 13219

Ministério da Saúde, Brasil. Carta de Ottawa. Carta De Ottawa (saude.gov.br)

Ministério das Relações Exteriores, Brasil. (2010). Promulga o Acordo Quadro entre o Governo da República Federativa do Brasil e o Governo do Canadá para Cooperação em Ciência, Tecnologia e Inovação. Base Legislação da Presidência da República - Decreto no 7.345 de 27 de outubro de 2010 (presidencia.gov.br)

Monteiro, L. Z., Varela, A. R., Alves, L. R., Santos, M. R. S., Lopes, G. R., Caetano Jr, M. A., \& Leandro, S. S. (2018). Prevalência e Fatores Associados ao Uso de Álcool e Tabaco em Universitários do Curso de Enfermagem. Rev. Eletr.Enferm, 20, a44. https://doi.org/10.5216/ree.v20.45296

Moryson, W., \& Stawińska-Witoszyńska, B. (2021). Excess Mortality of Males due to Malignant Lung Cancer in OECD Countries. Int. J. Environ. Res. Public Health, 18, 447. https://doi.org/10.3390/ijerph18020447

Munhoz, T. N., Santos, I. S., Nunes, B. P., Mola, C. L., Silva, I. C. M., \& Matijasevich, A. (2017). Tendências de Consumo Abusivo de Álcool nas Capitais Brasileiras entre os Anos de 2006 a 2013: Análise das Informações do VIGITEL. Cad Saude Publica, 33(7), e00104516. https://doi.org/10.1590/0102$311 \times 00104516$

Oliveira, M. S., Azambuja, A. P. R., \& Santos, A. P. (2015). Crenças Associadas ao Uso de Álcool em Populações Alcoolista e Não Alcoolista. Bol. Acad. Paul. Psicol., 35(88), 164-180. http://pepsic.bvsalud.org/scielo.php?script=sci_arttext\&pid=S1415-711X2015000100011\&lng=pt\&tlng=pt

Paillé, P., \& Mucchielli, A. (2016). L'Analyse Qualitative en Sciences Humaines et Sociales. (4a ed.), Armand Colin.

Pinheiro, M. A., Torres, L. F., Bezerra, M., Cavalcante, R. C., Alencar, R. D., Donato, A. C.,...Cavalcanti, L. P. G. (2017). Prevalência e Fatores Associados ao Consumo de Álcool e Tabaco entre Estudantes de Medicina no Nordeste do Brasil. Rev Bras Educ Med, 41(2), 231-250. https://doi.org/10.1590/1981$52712015 \mathrm{v} 41 \mathrm{n} 2 \mathrm{rb} 20160033$ 
Research, Society and Development, v. 10, n. 7, e29410716530, 2021

(CC BY 4.0) | ISSN 2525-3409 | DOI: http://dx.doi.org/10.33448/rsd-v10i7.16530

Rehm, J., \& Shield, K. D. (2021). Alcohol Use and Cancer in the European Union. Eur Addict Res, 27, 1-8. 10.1159/000507017

Sarich, P., Canfell, K., Egger, S., Banks, E., Joshy, G., Grogan, P., \& Weber, M. F. (2021). Alcohol Consumption, Drinking Patterns and Cancer Incidence in an Australian Cohort of 226,162 Participants Aged 45 years and over. Br J Cancer, 124, 513-523. https://doi.org/10.1038/s41416-020-01101-2

Silva, C. A. (2019). Inquérito Epidemiológico do Consumo do Álcool em Cortadores de Cana-de-Açúcar dos Estados da Paraíba e Goiás (Dissertação de mestrado). Universidade Federal de Goiás, Goiânia. (Disponível em: https://repositorio.bc.ufg.br/tede/handle/tede/9427)

Sue, V. M., \& Ritter, L. A. (2016). Sampling. In: V. M. Sue \& L. A. Ritter. Conducting online surveys. pp. 33-50. Thousand Oaks (CA): SAGE. https://dx.doi.org/10.4135/9781506335186

United Nations (n. d.). Ethnocultural characteristics. United Nations Statistics Division - Demographic and Social Statistics.

World Health Organization. (2018). Global Status Report on Alcohol and Health 2018. https://www.who.int/substance_abuse/pu blications/global _alcohol_report/en/

Zaitsu, M., Takeuchi, T., Kobayashi, Y., \& Kawachi, I. (2020). Light to Moderate Amount of Lifetime Alcohol Consumption and Risk of Cancer in Japan. Cancer, 126: 1031-1040. doi: 10.1002/cncr.32590.

Zanchetta, M., Kaszap, M., Monteiro, M., Gorospe IV, F., \& Pilon, R. (2011). Renewing perspectives on men's literacy on prostate cancer and engagement along the disease continuum. In: P. E. Spiess (Ed.). Prostate Cancer - Diagnostic and Therapeutic Advances. Rijeka: InTech, pp. 37-80. http://www.intechopen.com/articles/show/title/renewing-perspectives-on-men-s-prostate-cancer-literacy-and-engagement-along-the-disease-continuum.

Zanchetta, M., Cognet, M., Lam-Kin-Teng, M. R., Dumitriu, M. E., Haag, C., Kadio, B., \& Rénaud, L. (2018). French Media and Ideas about Prostate Cancer: Insights for Public Education. Health Promot Perspect, 8(2), 92-101. https://10.15171/hpp.208.12

Zanchetta, M., Maheu, C., Kolisnyk, O., Mohamed, M., Guruge, S., Kinslikh, D. \&Byam, A. (2017). Canadian Men's Self-Management of Chronic Diseases: A Literature Analysis of Strategies for Dealing with Risks and Promoting Wellness. Am J Mens Health, 11(4), 1077-1095. https://doi.org/10.1177/1557988315577674 